\title{
Design and Simulation of Low Noise Amplifier in Lower Frequency Peng Zhao ${ }^{1, a}$, Fan $\mathrm{Li}^{1}$, Jianhui Zhao ${ }^{1}$ and Jin Ding ${ }^{1}$ \\ ${ }^{1}$ BeiHang University, No. 37 Xueyuan Road, Haidian District, Beijing City, China \\ am15201275977@163.com
}

Keywords: low frequency; low noise amplifier; JFET; high gain.

\begin{abstract}
In order to amplify the output signal of an infrared detector, this paper analyzes the principle of infrared detector and noise characteristics of the amplifier circuit, and based on JFET devices and operational amplifiers a low frequency low noise amplifier is designed. The simulation shows that the gain is as high as $92 \mathrm{~dB}$ and the equivalent input noise voltage density within the main frequency is lower than $6.1 \mathrm{nV} \sqrt{ } \mathrm{Hz}$. This circuit is an ideal circuit of high gain and low noise for sensors with high output impedance especially for the infrared detector..
\end{abstract}

\section{Introduction}

Infrared detector is one of the fundamental parts of Infrared earth sensor which is the attitude measurement component on satellite [1]. To get the required signal, Infrared detector output which is weak electrical signal should be carried out after amplifying circuit and then processed by the subsequent processing circuit, which is shown in Fig. 1. For weak signals in amplitude of $\mu \mathrm{V}$, in addition to the external environmental disturbance, the inherent noise in the amplifier and amplifying circuit sometimes is much larger than the useful signal. Therefore, not only amplifying the signal, but also suppressing the inherent noise in the amplifier and amplifier circuit is very significant in order to get the useful signal. So in view of the weakness, the characteristic of low frequency of the infrared detector output signal, the low frequency low noise amplifier was designed and simulated.

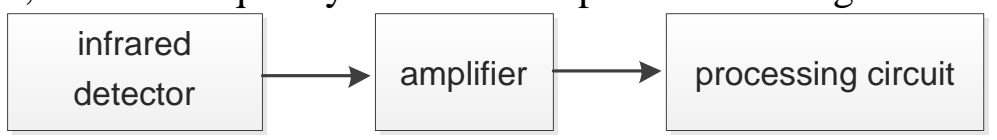

Fig. 1 The block diagram of infrared earth sensor composition

\section{The Principle of Infrared Detector}

The core of the NTC thermistor type infrared detector module is the NTC thermistor (negative temperature coefficient thermistor) the resistance of which declines with the increase of temperature. The detector is sealed with a metal shell, and the infrared radiation can penetrate the shell. There are two thermal sensors sealed in a metal casing. One component called primary is working in the normal way to accept the infrared radiation and the other one called patch which is sealing with silicone potting in order to avoid infrared irradiation is employed to temperature compensation. Two thermal sensors have the same parameters of thermal conductivity and are placed as nearly as possible, to ensure the same environmental conditions and good compensation effect.

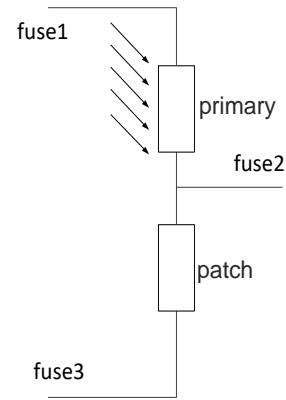

Fig. 2 Principle diagram of the infrared detector 
Temperature change rate type of general NTC thermistors is 3.5\% / K, which means that NTC thermistors are sensitive to the temperature change of $0.001 \mathrm{~K}$. At the room temperature, the resistance of commonly used NTC thermistors is about $280 \mathrm{k} \Omega$. As shown in Fig.2, assuming that power supply voltage between fuse 1 and fuse 3 is $5 \mathrm{~V}$, when there is a temperature change of $0.001^{\circ}$ $\mathrm{K}$, the primary's resistance value turns to $1-0.001 *(3.5 \%) * 280=279.9902 \mathrm{k} \Omega$ and output voltage of fuse 2 is $5 * 279.9901 /(279.9901+280)=2.49995625 \mathrm{~V}$, so that the change of fuse 2 output voltage is $2.5-2.49995625=43.75 * 10-6 \mathrm{~V}$, namely $43.75 \mathrm{u} \mathrm{V}$, which is a very weak signal. In this case, the role of the amplifier is to amplify the weak signal of the sensor output until the signal can be measured effectively.

Also due to the low scanning frequency (typical value $5 \mathrm{~Hz}-10 \mathrm{~Hz}$ ) of infrared earth sensor the frequency distribution of the output signal is in the low frequency band. Thus, it is necessary to design a low frequency low noise amplifier.

\section{Amplifier Noise Characteristics and Source}

There are several kinds of noise sources in the amplifier, such as thermal noise, shot noise, G-R noise and 1 / $\mathrm{f}$ noise. The 1 / $\mathrm{f}$ noise generally appears below $1 \mathrm{kHz}$ frequency, and G-R noise appears in the $1 \mathrm{kHz}$ to $100 \mathrm{kHz}$ frequency ranges. Due to the low-frequency distribution of the periodic wave signal of earth, the main noise sources of the low frequency low-noise amplifier are 1/f noise and G-R noise[2].

The amplifier circuit not only enlarges the output signal and the noise, but also enlarges its own ground noise. So the noise factor is put in place to specify the performance of an amplifier, with lower values indicating better performance.

Noise factor is described as the ratio of the output noise power of a device to the portion thereof attributable to thermal noise in the input termination at standard noise temperature (usually $290 \mathrm{~K}$ ). The noise factor is therefore the ratio of actual output noise to that which would remain if the device itself did not introduce noise, or the ratio of input SNR to output SNR.

$$
F=\frac{N_{o}}{N_{t o}}=\frac{E_{n i}^{2}}{E_{t}^{2}}
$$

To get a much lower noise factor, there are some matters that need attention.

One is noise matching. The noise source resistance of the amplifier is described as the ratio of noise voltage to the noise current. When the noise source resistance is equal to the resistance of the former level, it can achieve the minimum noise factor as showed in (2).

$$
F_{\text {opt }}=1+E_{n} I_{n} / 2 k T \Delta f
$$

At low frequency, when the resistance of the former level is small, it is recommended to use integrated operational amplifier or transistor which to design an amplifier. When the resistance of the former level is larger, it is better to use field-effect tube to get lower noise factor.

If several amplifiers are cascaded, the total noise factor of the amplifier structure is not so simple as the total gain of the amplifier structure which is equal to the product of all levels' gain. According to Friis' Formula in (3)::

$$
F=F_{1}+\frac{F_{2}-1}{K_{1}}+\frac{F_{3}-1}{K_{1} K_{2}}+\bullet \bullet+\frac{F_{n}-1}{K_{1} K_{2} \bullet \bullet \bullet K_{N-1}}
$$

Where $\mathrm{F}$ is the total noise factor, $\mathrm{Fn}$ is the noise factor of the $\mathrm{n}$-th device and $\mathrm{Kn}$ is the power gain of the n-th device.

In cascade amplifier circuit, the effect of different level on the total noise factor is different [2]. The first amplifier in a chain has the most significant effect on the total noise figure than any other amplifier in the chain. If the power gain of the first amplifier is much larger than others, the total noise factor is mainly affected by the first one. Thus, the lower noise factor amplifier should usually go first in a line of amplifiers. 


\section{The Design of The Low Frequency Low Noise Amplifier}

The amplifier circuit has three modules and is designed with cascade amplifiers. The composition of amplifier block diagram is shown in Fig.3 and the amplifier circuit principle diagram shown in Fig.4.

The junction field-effect transistors, which have high input impedance, low ground noise, a low noise factor, good thermal stability strong anti-interference ability[3], and an integrated operational amplifier are used for the first level to design an low-noise, high-gain preamplifier.

The second amplifying circuit is composed of an instrumentation amplifier structure. The instrumentation amplifier structure is a type of circuit that consists of three instrumentation amplifiers in a unique form. This circuit is features very low input offset voltage, low drift voltage, low-noise level, very high input impedance, super superior common mode rejection ratio, low-noise and wide gain bandwidth. Finally, after amplification it has an output buffer circuit to reduce the output impedance[4-5].

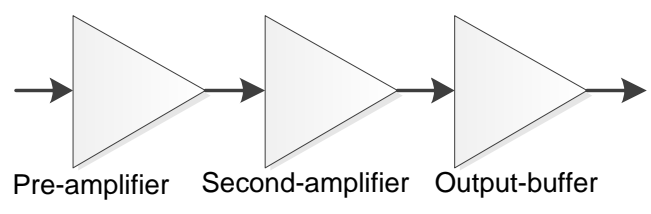

Fig. 3 Amplifier design block diagram

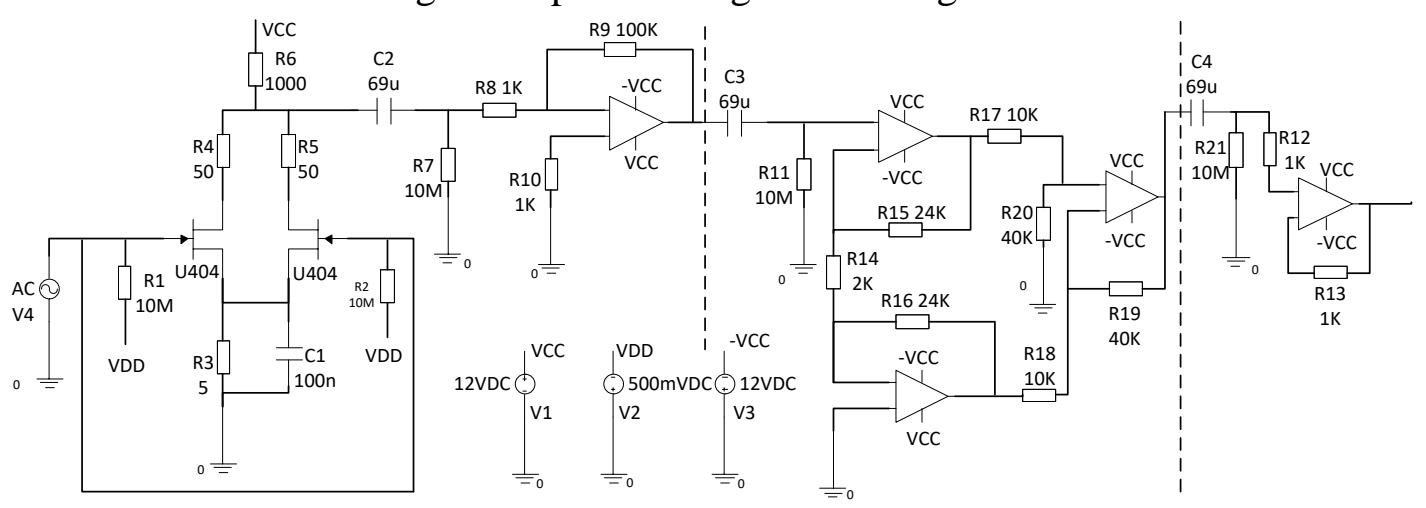

Fig. 4 Amplifier circuit principle diagram

\section{Pre-amplifier}

Through DC filter, the input signal gets to the differential amplifier circuit. Differential amplifier circuit is made up of two JFETs which have the same characteristic parameters using common source structure. The rest of the circuit parameters in Fig. 4 are $\mathrm{R} 4=\mathrm{R} 5=50 \Omega, \mathrm{R} 1=\mathrm{R} 2=10 \mathrm{M} \Omega, \mathrm{R} 6=1 \mathrm{k} \Omega$, which is a completely symmetrical structure. The general form of the differential amplifier circuit is that it only selects one end of the symmetric structure as output, and the other end as output reference end to suppress the temperature drift. But this design connects the two ends of JFETs to get the output signal unlike the general way, which can increase the anti-interference ability of the circuit, make the output noise offset each other, and improve the noise performance.

The output signal of the differential circuit is phase reversal with the original. And there is subsequent circuit shown inFig.4 which is an inverting amplifier using AD743 to reverse the phase as well as further amplify the signal.

\section{Second-level Amplifier}

The AD743 is an ultralow noise, precision, FET input, monolithic operational amplifier. It offers a combination of the ultralow voltage noise generally associated with bipolar input op amps and the very low input current of a FET input device. Thus A1, A2, A3 are all AD743 op-amps.

Circuit structure is in pseudo-differential input mode that one noninverting input is connected to the input signal while the other noninverting input is connected to the signal ground. Pseudo differential input mode reduces the potential influence caused by the different between signal source and equipment reference of ground, improves the accuracy of measurement, reduces the noise 
introduced by ground potential changes, suppress the temperature drift at the same time, and improve the noise performance of the circuit.

The input signal in $\mu \mathrm{V}$ level has been amplified almost 400 times by the preamplifier so that in this circuit level, the power gain is 100 times. And the selection of resistance for R15 and R16 to $24 \mathrm{k} \Omega$, R17 and R18 to $10 \mathrm{k} \Omega, \mathrm{R} 19$ and R20 to $40 \mathrm{k} \Omega, \mathrm{R} 14$ to $2 \mathrm{k} \Omega$. Circuit diagram is shown in Fig. 4.

\section{Output Buffer}

The next level shown in Fig.8 is a voltage follower an op-amp circuit which has a voltage gain of 1. The output voltage directly follows input voltage, which means the output voltage is the same as the input voltage. A voltage follower just acts as a buffer, providing no amplification or attenuation to the input signal but a very high input impedance.

\section{Simulation Results}

The amplifier circuit is simulated through software Pspice 16.5.As showed in Table 1, the equivalent input noise voltage density changes in power attenuation with the increase of frequency. When the frequency is greater than $10 \mathrm{~Hz}$, the density is less than $6.1 \mathrm{nV} \sqrt{\mathrm{Hz}}$ and the gain is up to $92 \mathrm{~dB}$ while the bandwidth is between $1 \mathrm{~Hz}$ and $100 \mathrm{kHz}$.

Table 1 Simulation results

\begin{tabular}{ccc}
\hline Frequency $[\mathrm{Hz}]$ & Noise $[\mathrm{nV} \sqrt{\mathrm{Hz}}]$ & Gain[dB] \\
\hline 0.1 & 59.2 & 70.6 \\
1 & 18.7 & 89.1 \\
10 & 6.1 & 92.0 \\
100 & 2.4 & 92.0 \\
1000 & 1.6 & 92.0 \\
10000 & 1.5 & 92.0 \\
100000 & 1.5 & 89.3 \\
1000000 & 1.9 & 18.0 \\
\hline
\end{tabular}

\section{Summary}

Since the low amplitude output signal of the infrared detector is with low frequency and high source resistance, a low frequency low noise amplifier is designed and analyzed with simulation. The low amplitude signal is amplified by the differential JFET circuit with little noise, and then amplified by the intermediate amplifier circuit which is output by a buffer stage. The simulation results demonstrate that the output gain can reach $92 \mathrm{~dB}$ with $1 \mathrm{~Hz} \sim 10 \mathrm{k} \mathrm{Hz}$ signal and when the frequency is larger than $10 \mathrm{~Hz}$, the voltage density of equivalent input noise is inferior to $6.1 \mathrm{nV} \sqrt{\mathrm{Hz}}$. The designed amplifier could magnify the output signal of the infrared detector with a little noise.

\section{References}

[1] Meng H. A Low Frequency Noise Test System for Thermistor Infrared Detectors[J]. Computer Measurement \& Control, 2012, 2: 009.

[2] Gao J Z. Detection of weak signals[J]. 2004.

[3] Zhou YY. Application Research of Low Frequency Noise Amplifier in Low Frequency[J]. Journal of hubei university of technology, 2006, 21 (5) : 42-44.(In Chinese)

[4] LI Y, GUO S, GAO F. Design and simulation of low-noise amplifier for detection of 1/f electrical noise[J]. Modern Electronics Technique, 2015, 4: 022.

[5] Xiaofei Z, Haobin D, Yongkang L. Design of JFET Low Noise Preamplifier[J]. Chinese Journal of Engineering Geophysics, 2009, 3: 026. 